\title{
A Bayesian Approach to Characterize Hong Kong Influenza Surveillance Systems
}

\author{
Ying Zhang*, Ali Arab and Michael A. Stoto \\ Georgetown University, Washington, DC, USA
}

\section{Objective}

Our goal is to develop a statistical model for characterizing influenza surveillance systems that will be helpful in interpreting multiple streams of influenza surveillance data in future outbreaks.

\section{Introduction}

Syndromic surveillance has been widely used in influenza surveillance worldwide. However, despite the potential benefits created by the large volume of data, biases due to the changes in healthcare seeking behavior and physicians' reporting behavior, as well as the background noise caused by seasonal flu epidemics, contribute to the complexity of the surveillance system and may limit its utility as a tool for early detection [1,2]. Since most current analysis methods are developed for outbreak detection, there are few tools to characterize influenza surveillance data for situational awareness purposes in a quantitative manner.

Hong Kong Centre for Health Protection (CHP) has a comprehensive influenza surveillance system based on healthcare providers, laboratories, schools, daycare centers and residential care homes for the elderly. Hong Kong usually experiences a summer peak in July and August [3], which potentially doubles the data volume and constitutes a natural experiment to assess the effect of school-age children in the influenza transmission dynamics. The richness of the available data and the unique epidemiological characteristics make Hong Kong an ideal study object to develop and evaluate our model.

\section{Methods}

We have constructed a Bayesian statistical model for influenza surveillance data by parameterizing factors that describe disease transmission, behavior patterns in health care seeking and provision, and biases and errors embedded in the reporting process (Figure 1). The prior distributions are selected for each of the parameters to reflect knowledge of influenza epidemiology and the likely biases in each data system. Using the Markov Chain Monte-Carlo (MCMC) method in OpenBUGS, a posterior distribution can be generated for every parameter to characterize each data stream. The ratios of specific pairs of data streams are assessed in order to identify patterns in the change of ratios at different stage of the flu season.

\section{Results}

Preliminary results, as shown in Figure 2, incorporate confirmed influenza infection (solid line), influenza-like illness (double solid line), fever cases (dashed line), and Google search index (round dashed line). Although most of these data series track together, differences among them suggest reporting bias related to public awareness, which will be addressed in the statistical modeling.

\section{Conclusions}

The posterior distribution for parameters and ratios between individual data streams can be used to characterize influenza surveillance systems in terms of tendency in peak early or late, or to over or under represent actual influenza cases. To better interpret syndromic surveillance data for situational awareness purposes, behavioral data related to healthcare resource utilization, such as the percentage of intended GP visit among people with ILI, need to be collected together with the flu activity surveillance.

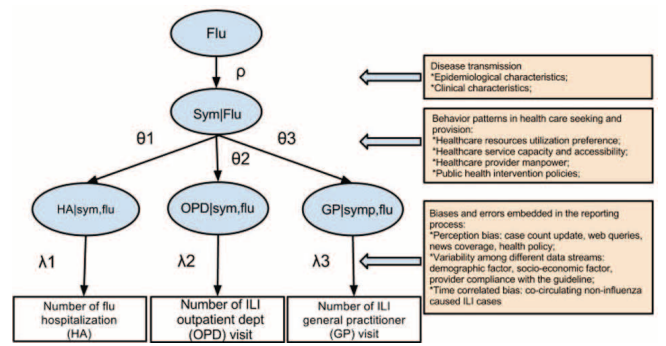

Conceptual model for influenza surveillance statistical model Blue circles: unobservable true value; white boxes: observation; orange boxes: factors

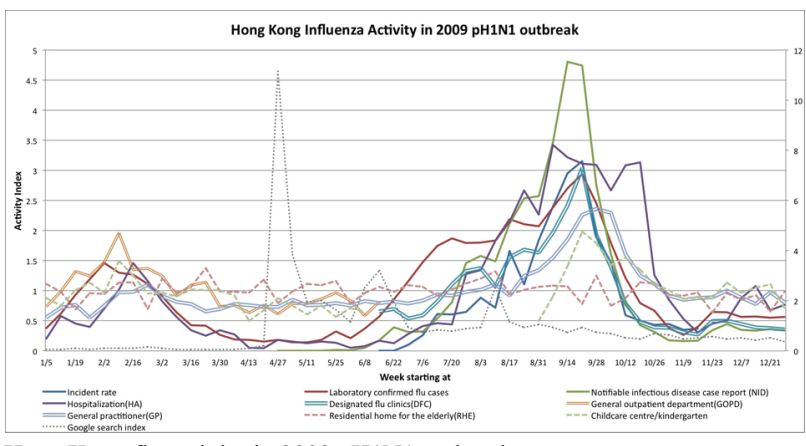

Hong Kong flu activity in 2009 pH1N1 outbreak

\section{Keywords}

situational awareness; modeling; epidemiology; influenza surveillance; Bayesian

\section{Acknowledgments}

CDC, Hong Kong University

Center for Health Protection, Hong Kong SAR

\section{References}

1.Zhang, Y., May, L., \& Stoto, M. A. (2011). Evaluating syndromic surveillance systems at institutions of higher education (IHEs): A retrospective analysis of the $2009 \mathrm{H} 1 \mathrm{~N} 1$ influenza pandemic at two universities. BMC Public Health, 11, 591.

2. Stoto MA (2012) The Effectiveness of U.S. Public Health Surveillance Systems for Situational Awareness during the 2009 H1N1 Pandemic: A Retrospective Analysis. PLoS ONE 7(8): e40984.

3. Chan, P. K. S., Mok, H. Y., Lee, T. C., Chu, I. M. T., Lam, W., \& Sung, J. J. Y. (2009). Seasonal influenza activity in Hong Kong and its association with meteorological variations. Journal of Medical Virology, 81(10), 1797-1806.

\footnotetext{
*Ying Zhang

E-mail: yz62@georgetown.edu
} 